\title{
Accumulated Gouy phase shift in Gaussian beam propagation through first-order optical systems
}

\author{
M. Fatih Erden and Haldun M. Ozaktas \\ Department of Electrical Engineering, Bilkent University, 06533 Bilkent, Ankara, Turkey
}

Received August 6, 1996; revised manuscript received January 24, 1997; accepted February 18, 1997

\begin{abstract}
We define the accumulated Gouy phase shift as the on-axis phase accumulated by a Gaussian beam in passing through an optical system, in excess of the phase accumulated by a plane wave. We give an expression for the accumulated Gouy phase shift in terms of the parameters of the system through which the beam propagates. This quantity complements the beam diameter and the wave-front radius of curvature to constitute three parameters that uniquely characterize the beam with respect to a reference point in the system. Measurement of these parameters allows one to uniquely recover the parameters characterizing the first-order system through which the beam propagates. (C) 1997 Optical Society of America [S0740-3232(97)01408-7]
\end{abstract}

\section{INTRODUCTION}

A paraxial wave ${ }^{1}$ is a plane wave $\exp (i k z)$ modulated by a complex envelope $A(x, y, z)$ that is assumed to be a slowly varying function of position. In paraxial waves, in order for the complex amplitude $U(x, y, z)$, expressed as

$$
U(x, y, z)=A(x, y, z) \exp (i k z),
$$

to satisfy Helmholtz equation, $A(x, y, z)$ must satisfy the paraxial Helmholtz equation. One of the solutions of the paraxial Helmholtz equation is a Gaussian beam, for which we can express the complex envelope $A(x, y, z)$ as

$$
\begin{aligned}
A(x, y, z) & =\frac{A_{1}}{q(z)} \exp \left[i k\left(x^{2}+y^{2}\right) / 2 q(z)\right], \\
q(z) & =z-i z_{0},
\end{aligned}
$$

where $z_{0}$ is known as the Rayleigh range and $A_{1}$ is an arbitrary complex constant. We can also express $q(z)$ through

$$
\frac{1}{q(z)}=\frac{1}{r(z)}+i \frac{\lambda}{\pi w^{2}(z)},
$$

where $w(z)$ and $r(z)$ are the beam width and the wavefront radius of curvature, respectively, of Gaussian beams. With these newly defined parameters, the expression in Eq. (2) becomes

$$
\begin{aligned}
A(x, y, z)= & A_{0} \frac{w_{0}}{w(z)} \exp \left[-\frac{x^{2}+y^{2}}{w^{2}(z)}\right] \exp \left[i \pi \frac{x^{2}+y^{2}}{\lambda r(z)}\right] \\
& \times \exp [-i \zeta(z)],
\end{aligned}
$$

where $A_{0}=-A_{1} / i z_{0}$ is another complex constant and

$$
\begin{gathered}
w(z)=w_{0} \sqrt{1+z^{2} / z_{0}^{2}}, \quad r(z)=z\left(1+z_{0}^{2} / z^{2}\right), \\
\zeta(z)=\tan ^{-1} z / z_{0}, \quad w_{0}=\sqrt{\lambda z_{0} / \pi} .
\end{gathered}
$$

In these equations, $w_{0}$ and $\zeta(z)$ are defined as the beamwaist diameter and the Gouy phase shift, respectively.
These expressions assume that the beam waist is located at $z=0$, but they can be generalized to any waist location at $z=z_{w}$ by simply replacing $z$ with $z-z_{w}$.

In this paper we consider Gaussian beams passing through centered, axially symmetric quadratic-phase optical systems under the standard approximations of Fourier optics. ${ }^{1}$ Thin lenses, arbitrary sections of free space (under the Fresnel approximation), quadratic gradedindex media, and any combinations of these belong to the class of quadratic-phase systems. We characterize the members of quadratic-phase systems through ${ }^{2-6}$

$$
\begin{aligned}
p_{\text {out }}(x) & =\int_{-\infty}^{\infty} h\left(x, x^{\prime}\right) p_{\text {in }}\left(x^{\prime}\right) \mathrm{d} x^{\prime}, \\
h\left(x, x^{\prime}\right) & =K \exp \left[i \pi\left(\alpha x^{2}-2 \beta x x^{\prime}+\gamma x^{\prime 2}\right)\right],
\end{aligned}
$$

where $K$ is a complex constant and $\alpha, \beta$, and $\gamma$ are real constants. Thus, apart from the constant factor $K$, which has no effect on the resulting spatial distribution, a member of the class of quadratic-phase systems is completely specified by the three parameters $\alpha, \beta$, and $\gamma$. Alternatively, such a system can also be completely specified by the transformation matrix ${ }^{2-6}$

$$
\mathbf{T} \equiv\left[\begin{array}{ll}
A & B \\
C & D
\end{array}\right] \equiv\left[\begin{array}{cc}
\gamma / \beta & 1 / \beta \\
-\beta+\alpha \gamma / \beta & \alpha / \beta
\end{array}\right],
$$

with unity determinant (i.e., $A D-B C=1$ ). If several systems each characterized by such a matrix are cascaded, the matrix characterizing the overall system can be found by multiplying the matrices of the several systems. ${ }^{2,4,5}$ The matrix defined above also corresponds to the well-known ray matrix employed in ray optical analysis. 1,3

If we want to express the transformation in Eq. (6) in terms of the matrix elements $A B C D$ of the medium, it turns out that ${ }^{5,7}$ 


$$
\begin{aligned}
p_{\text {out }}(x) & =\exp \left(i k L_{0}\right) \int_{-\infty}^{\infty} \tilde{h}\left(x, x^{\prime}\right) p_{\text {in }}\left(x^{\prime}\right) \mathrm{d} x^{\prime}, \\
\tilde{h}\left(x, x^{\prime}\right) & =\frac{1}{\sqrt{i B}} \exp \left[\frac{i \pi}{B}\left(D x^{2}-2 x x^{\prime}+A x^{\prime 2}\right)\right] .
\end{aligned}
$$

In this equation, $L_{0}$ is the on-axis optical length of the medium. ${ }^{7}$ For two-dimensional, centered, and axially symmetric systems this transformation becomes

$$
\begin{aligned}
p_{\text {out }}(x, y)= & \exp \left(i k L_{0}\right) \int_{-\infty}^{\infty} \int_{-\infty}^{\infty} \tilde{h}\left(x, x^{\prime}\right) \tilde{h}\left(y, y^{\prime}\right) \\
& \times p_{\text {in }}\left(x^{\prime}, y^{\prime}\right) \mathrm{d} x^{\prime} \mathrm{d} y^{\prime} .
\end{aligned}
$$

In this paper we define the accumulated Gouy phase shift and give an expression for it in terms of the transformation matrix elements $A B C D$ of the medium. We observe that this quantity complements the beam diameter and the wave-front radius of curvature to constitute three parameters that uniquely characterize the first-order optical system parameters through which the beam propagates.

\section{ACCUMULATED GOUY PHASE SHIFT}

\section{A. Definition}

The Gouy phase shift defined in Eq. (5) is the on-axis phase of a Gaussian beam with respect to the beam waist in excess of the phase of a plane wave $\exp (i k z)$. It is not independent of beam diameter $w$ and the wave-front radius of curvature $r$. That is, if we know $w$ and $r$ of a beam at a specific plane, we can also obtain the Gouy phase shift at that plane. More specifically, from Eq. (5) we can find

$$
\zeta(z)=\tan ^{-1} \frac{\pi w^{2}(z)}{\lambda r(z)} .
$$

When a Gaussian beam propagates through an optical system composed of several lenses, there may be a number of beam waists. In this case it does not seem possible to interpret the Gouy phase shift as defined above in a meaningful manner. It may be possible to interpret $\zeta$ as the on-axis phase of the beam in excess of that of a plane wave with respect to the last (possibly virtual) waist. Of greater interest would be the phase shift accumulated by the beam as it passes through several lenses and sections of free space with respect to a single reference point in the system. Thus we define the accumulated Gouy phase shift $\tilde{\zeta}$ of a Gaussian beam passing through an optical system as the on-axis phase accumulated by the beam in excess of the factor $\exp \left(i k L_{0}\right)$ in Eq. (9) (this factor can also be thought as the on-axis phase that would be accumulated by a plane wave). Mathematically,

$$
-\tilde{\zeta}=\arg \left\{p_{\text {out }}(0,0)\right\}-\arg \left\{p_{\text {in }}(0,0)\right\}-k L_{0},
$$

where $p_{\text {in }}(x, y)$ and $p_{\text {out }}(x, y)$ denote the input and the output Gaussian beams, respectively, and $\arg \{\cdot\}$ denotes the argument (phase).

In Ref. 8, transformation of the generalized beam-mode parameters is analyzed with operator algebra. The out- put parameters are related to the input parameters through transformation matrix elements $A B C D$ of the medium. When the matrix elements are real, as is the case here, the generalized beams correspond to conventional Hermite-Gaussian beams, the lowest order of which is the Gaussian beam.

Let us now consider a Gaussian beam with parameters $w_{\text {in }}$ and $r_{\text {in }}$ input to a quadratic-phase system, and let the system be characterized by the transformation matrix with elements $A B C D$. The output beam parameters are $w_{\text {out }}$ and $r_{\text {out }}$. Let us define the complex parameters $q_{\text {in }}$ and $q_{\text {out }}$ associated with the input and the output Gaussian beams, respectively, through Eq. (3). We know that $q_{\text {out }}$ is related to $q_{\text {in }}$ through matrix elements $A B C D$ as $^{1,7,8}$

$$
\lambda q_{\text {out }}=\frac{A \lambda q_{\text {in }}+B}{C \lambda q_{\text {in }}+D} .
$$

Using this expression, we can relate the output parameters $w_{\text {out }}$ and $r_{\text {out }}$ to the input parameters $w_{\text {in }}$ and $r_{\text {in }}$ through $A B C D$ as

$$
\begin{aligned}
w_{\text {out }}^{2} & =w_{\text {in }}^{2}\left(A+\frac{B}{\lambda r_{\text {in }}}\right)^{2}+\frac{B^{2}}{\pi^{2} w_{\text {in }}^{2}}, \\
\frac{1}{r_{\text {out }}} & =\frac{\left(\lambda C+\frac{D}{r_{\text {in }}}\right)\left(A+\frac{B}{\lambda r_{\text {in }}}\right)+\frac{B D \lambda}{\pi^{2} w_{\text {in }}^{4}}}{\left(A+\frac{B}{\lambda r_{\text {in }}}\right)^{2}+\frac{B^{2}}{\pi^{2} w_{\text {in }}^{4}}} .
\end{aligned}
$$

In this paper we show that the accumulated Gouy phase shift $\tilde{\zeta}$ can be similarly expressed as

$$
\tan \tilde{\zeta}=\frac{B}{\left(A+\frac{B}{\lambda r_{\text {in }}}\right) \pi w_{\text {in }}^{2}} .
$$

Two proofs of this expression are given in Section 3. The first one is algebraically straightforward, but the second one although somewhat long, is more instructive.

As a result, if we consider a Gaussian beam with parameters $w_{\text {in }}$ and $r_{\text {in }}$ input to a quadratic-phase system characterized by the matrix with elements $A B C D$, the parameters $w_{\text {out }}$ and $r_{\text {out }}$ of the output beam as well as the accumulated Gouy phase shift from input to output can be found from Eqs. (13)-(15). Notice that the accumulated Gouy phase shift depends on only two of the three independent parameters that characterize the first-order system. Thus two systems that have the same values of $A$ and $B$ but different values of $C$ and $D$ may have the same accumulated Gouy phase shift.

\section{B. Interpretation As an Independent Parameter}

We may also look at the problem the other way around. Let us assume that we know the parameters $w_{\text {in }}$ and $r_{\text {in }}$ of the Gaussian beam at the input and that we can measure $w_{\text {out }}$ and $r_{\text {out }}$ at the output of the quadratic-phase system, and let us assume that we also know the accumulated Gouy phase shift between the input and the output planes (if we know the on-axis optical length of the system, we can obtain both the output wave-front radius of curvature and the accumulated Gouy phase shift by interfering the 
beam with a plane). Then we can recover the matrix elements $A B C D$ of the system by using Eqs. (13)-(15) and the identity $A D-B C=1$ as

$$
\begin{aligned}
& A=\frac{w_{\text {out }}}{w_{\text {in }}} \cos \tilde{\zeta}-\frac{\pi w_{\text {in }} w_{\text {out }} \sin \tilde{\zeta}}{\lambda r_{\text {in }}}, \\
& B=\pi w_{\text {in }} w_{\text {out }} \sin \tilde{\zeta}, \\
& C=\frac{A}{\lambda r_{\text {out }}}-\frac{\frac{B}{\pi^{2} w_{\text {in }}^{4}}+\frac{1}{\lambda r_{\text {in }}}\left(A+\frac{B}{\lambda r_{\text {in }}}\right)}{\left(A+\frac{B}{\lambda r_{\text {in }}}\right)^{2}+\frac{B^{2}}{\pi^{2} w_{\text {in }}^{4}}}, \\
& D=\frac{1+B C}{A} .
\end{aligned}
$$

Notice that the $A B C D$ parameters of the system could not be recovered from measurements of a single Gaussian probe by employing the conventional Gouy phase shift [Eq. (10)]. This is because the conventional Gouy phase shift is not independent of beam diameter $w$ and the wave-front radius of curvature $r$. In contrast, the accumulated Gouy phase shift is an independent parameter that complements the beam diameter and the wave-front radius of curvature to constitute three parameters that uniquely characterize the beam with respect to a reference point in the system. This means that knowledge of these three parameters at any single plane in the system allows them to be calculated at any other plane in the system. Measurement of these parameters allows one to uniquely recover the parameters that completely characterize the quadratic-phase system through which the beam propagates. Remember that such systems are characterized by three parameters $(\alpha, \beta, \gamma$, or any three of $A B C D)$. Thus we would expect the effect of such a system on a beam passing through it also to be characterized by three parameters. The conventional Gouy phase shift, which is not independent of $w$ and $r$ and is difficult to interpret meaningfully in multilens systems, cannot serve as a third parameter. This was the motivation behind defining the accumulated Gouy phase shift. The importance of this quantity is further supported by its direct relation to the fractional-Fourier-transform parameter, ${ }^{3,9,10}$ as discussed at length elsewhere. ${ }^{11}$

\section{PROOF OF THE ACCUMULATED GOUY PHASE-SHIFT EXPRESSION}

In this section we give two proofs for the expression in Eq. (15).

\section{A. Proof A}

The output Gaussian beam $p_{\text {out }}(x, y)$ is related to the input Gaussian beam $p_{\text {in }}\left(x^{\prime}, y^{\prime}\right)$ through Eq. (9) as

$$
\begin{aligned}
p_{\text {out }}(x, y)= & \exp \left(i k L_{0}\right) \int_{-\infty}^{\infty} \int_{-\infty}^{\infty} \tilde{h}\left(x, x^{\prime}\right) \tilde{h}\left(y, y^{\prime}\right) \\
& \times p_{\text {in }}\left(x^{\prime}, y^{\prime}\right) \mathrm{d} x^{\prime} \mathrm{d} y^{\prime},
\end{aligned}
$$

where $\tilde{h}(\cdot, \cdot)$ is the kernel in Eq. (8) and

$$
p_{\text {in }}(x, y)=A_{\text {in }} \frac{w_{0}}{w_{\text {in }}} \exp \left(-\frac{x^{2}+y^{2}}{w_{\text {in }}^{2}}\right) \exp \left(i \pi \frac{x^{2}+y^{2}}{\lambda r_{\text {in }}}\right)
$$

We know from Ref. 7 that

$$
\int_{-\infty}^{\infty} \exp \left(-x^{2} r^{2}-q x\right) \mathrm{d} x=\frac{\sqrt{\pi}}{r} \exp \left(\frac{q^{2}}{4 r^{2}}\right) .
$$

Using this identity, after some algebra we obtain $p_{\text {out }}(x, y)$ as

$$
\begin{aligned}
p_{\text {out }}(x, y)= & A_{\text {in }} \frac{w_{0}}{w_{\text {out }}} \exp \left(i k L_{0}\right) \exp \left[-\frac{x^{2}+y^{2}}{w_{\text {out }}^{2}}\right] \\
& \times \exp \left[i \pi \frac{x^{2}+y^{2}}{\lambda r_{\text {out }}}\right] \exp (-i \tilde{\zeta}),
\end{aligned}
$$

where

$$
\begin{aligned}
w_{\text {out }}^{2} & =w_{\text {in }}^{2}\left(A+\frac{B}{\lambda r_{\text {in }}}\right)^{2}+\frac{B^{2}}{\pi^{2} w_{\text {in }}^{2}}, \\
\frac{1}{r_{\text {out }}} & =\frac{\left(\lambda C+\frac{D}{r_{\text {in }}}\right)\left(A+\frac{B}{\lambda r_{\text {in }}}\right)+\frac{B D \lambda}{\pi^{2} w_{\text {in }}^{4}}}{\left(A+\frac{B}{\lambda r_{\text {in }}}\right)^{2}+\frac{B^{2}}{\pi^{2} w_{\text {in }}^{4}}} \\
\tan \tilde{\zeta} & =\frac{B}{\left(A+\frac{B}{\lambda r_{\text {in }}}\right) \pi w_{\text {in }}^{2}} .
\end{aligned}
$$

\section{B. Proof B}

Lemma 1: The expression in Eq. (15) is consistent under concatenation [i.e., if two systems for which Eq. (15) holds are cascaded, the resultant accumulated Gouy phase shift is also in the form of Eq. (15) and is expressed as the summation of the phase shifts associated with the individual systems].

Proof: Suppose that we have two systems with transformation matrix elements $A_{1} B_{1} C_{1} D_{1}$ and $A_{2} B_{2} C_{2} D_{2}$ concatenated one after another. Let us call the Gaussian beam parameters at the input of the first system $w_{\text {in } 1}$ and $r_{\text {in } 1}$, at the output of the first system $w_{\text {out1 }}=w_{\text {in2 }}$ and $r_{\text {out1 }}=r_{\text {in2 }}$ (which are also the input parameters of the second system), and at the output of the second system $w_{\text {out2 }}$ and $r_{\text {out } 2}$. We also have the accumulated Gouy phase shifts $\widetilde{\zeta}_{1}$ and $\widetilde{\zeta}_{2}$ associated with the first and second systems, respectively. Let us also assume that both systems satisfy Eq. (15). Then

$$
\begin{aligned}
\tan \tilde{\zeta}_{1} & =\frac{B_{1}}{\left(A_{1}+\frac{B_{1}}{\lambda r_{\text {in } 1}}\right) \pi w_{\text {in } 1}^{2}}, \\
\tan \tilde{\zeta}_{2} & =\frac{B_{2}}{\left(A_{2}+\frac{B_{2}}{\lambda r_{\text {out } 1}}\right) \pi w_{\text {out } 1}^{2}} .
\end{aligned}
$$

We can obtain $w_{\text {out1 }}$ and $r_{\text {out1 }}$ used in Eq. (28) in terms of $w_{\text {in }}, r_{\text {in }}$ and the matrix elements of the first system 
through Eqs. (13) and (14). We also have the overall system transformation matrix elements $A B C D$ through

$$
\left[\begin{array}{ll}
A & B \\
C & D
\end{array}\right]=\left[\begin{array}{ll}
A_{2} & B_{2} \\
C_{2} & D_{2}
\end{array}\right]\left[\begin{array}{cc}
A_{1} & B_{1} \\
C_{1} & D_{1}
\end{array}\right]
$$

We have all the necessary equations, so although the computations are somewhat long, it is just a matter of straightforward algebra to show that

$$
\tilde{\zeta}_{1}+\tilde{\zeta}_{2}=\tilde{\zeta}_{r}=\tan ^{-1} \frac{B}{\left(A+\frac{B}{\lambda r_{\mathrm{in} 1}}\right) \pi w_{\mathrm{in} 1}^{2}},
$$

where $\tilde{\zeta}_{r}$ is the accumulated Gouy phase shift associated with the cascaded system.

Lemma 2: Equation (15) holds for systems with upper triangular matrices of the form

$$
\mathbf{T} \equiv\left[\begin{array}{cc}
1 & \lambda d \\
0 & 1
\end{array}\right]
$$

(The matrix associated with a section of free space of length $d$ is of this form.)

Proof: Let us consider a Gaussian beam with $w_{\text {in }}$ and $r_{\text {in }}$ input to a system. With these parameters, the complex envelope of the input Gaussian beam defined in Eq. (2) becomes

$$
A_{\text {in }}(x, y)=A_{0} \frac{w_{0}}{w_{\text {in }}} \exp \left(-\frac{x^{2}+y^{2}}{w_{\text {in }}^{2}}\right) \exp \left(i \pi \frac{x^{2}+y^{2}}{\lambda r_{\text {in }}}\right) .
$$

After taking the Fresnel integral of this expression, we find the output Gaussian beam as

$$
\begin{aligned}
A_{\text {out }}(x, y)= & A_{0} \frac{w_{0}}{w_{\text {out }}} \exp \left(-\frac{x^{2}+y^{2}}{w_{\text {out }}^{2}}\right) \exp \left(i \pi \frac{x^{2}+y^{2}}{\lambda r_{\text {out }}}\right) \\
& \times \exp (-i \tilde{\zeta}),
\end{aligned}
$$

where $w_{\text {out }}$ and $r_{\text {out }}$ in Eq. (33) are in the form of Eqs. (13) and (14) with $A B C D$ as the parameters of the matrix in Eq. (31) and

$$
\tan \tilde{\zeta}=\frac{\lambda d}{\left(1+\frac{d}{r_{\text {in }}}\right) \pi w_{\text {in }}^{2}} .
$$

We see that this expression is consistent with the one in Eq. (15) with $A=1$ and $B=\lambda d$, as is the case for the matrix in Eq. (31).

Lemma 3: Equation (15) holds for systems with lower triangular matrices of the form

$$
\mathbf{T}=\left[\begin{array}{cc}
1 & 0 \\
\frac{-1}{\lambda f} & 1
\end{array}\right]
$$

(The matrix associated with a thin lens of focal length $f$ is of this form.)

Proof: Again starting with the complex-envelope expression of the input Gaussian beam in Eq. (32), we know that after passage through a lens of focal length $f$, this complex envelope becomes

$$
\begin{aligned}
A_{\text {out }}(x, y)= & A_{0} \frac{w_{0}}{w_{\text {in }}} \exp \left(-\frac{x^{2}+y^{2}}{w_{\text {in }}^{2}}\right) \exp \left(i \pi \frac{x^{2}+y^{2}}{\lambda r_{\text {in }}}\right) \\
& \times \exp \left(-i \pi \frac{x^{2}}{\lambda f}\right) .
\end{aligned}
$$

Observing Eqs. (32) and (36), we see that when a Gaussian beam passes through a lens, its wave-front radius of curvature $r_{\text {out }}$ will be the only parameter that changes according to $r_{\text {out }}^{-1}=r_{\text {in }}^{-1}-f^{-1}$, whereas the beam diameter remains unchanged and there is no accumulated Gouy phase shift $(\tilde{\zeta}=0)$. This result is consistent with Eq. (15), as we can again obtain $\tilde{\zeta}=0$ from this equation together with the transformation matrix expressed in Eq. (35).

Proof of equation 15: Any $2 \times 2$ matrix of unity determinant can be decomposed as

$$
\mathbf{T} \equiv\left[\begin{array}{ll}
A & B \\
C & D
\end{array}\right] \equiv\left[\begin{array}{cc}
1 & (A-1) / C \\
0 & 1
\end{array}\right]\left[\begin{array}{cc}
1 & 0 \\
C & 1
\end{array}\right]\left[\begin{array}{cc}
1 & (D-1) / C \\
0 & 1
\end{array}\right]
$$

Thus any quadratic-phase system can be modeled as two sections of free space with a lens in between. We have shown in lemmas 2, 3 that the expression in Eq. (15) holds for both lenses and free-space sections. Then with the help of lemma 1 we can say that the accumulated Gouy phase-shift expression in Eq. (15) also holds for the cascaded system in Eq. (37) and hence for quadraticphase systems.

\section{CONCLUSION}

In this paper we defined the accumulated Gouy phase shift, and gave an expression for it in terms of the transformation matrix elements of the system through which the beam propagates. We observed that this quantity is independent of beam diameter $w$ and the wave-front radius of curvature $r$. Thus, measurement of the accumulated Gouy phase shift, the beam diameter, and the wavefront radius of curvature allows one to uniquely recover the parameters that completely characterize the quadratic-phase system through which the beam propagates.

\section{REFERENCES}

1. B. E. A. Saleh and M. C. Teich, Fundamentals of Photonics (Wiley, New York, 1991).

2. M. J. Bastiaans, "Wigner distribution function and its application to first-order optics," J. Opt. Soc. Am. 69, 17101716 (1979).

3. H. M. Ozaktas and D. Mendlovic, "Fractional Fourier optics," J. Opt. Soc. Am. A 12, 743-751 (1995).

4. K. B. Wolf, Integral Transforms in Science and Engineering (Plenum, New York, 1979).

5. M. Nazarathy and J. Shamir, "First-order optics-a canonical operator representation: lossless systems," J. Opt. Soc. Am. 72, 356-364 (1982).

6. S. Abe and J. T. Sheridan, "Optical operations on wave functions as the Abelian subgroups of the special affine Fourier transformation," Opt. Lett. 19, 1801-1803 (1994).

7. A. E. Siegman, Lasers (University Science Books, Mill Valley, Calif., 1986).

8. M. Nazarathy, A. Hardy, and J. Shamir, "Generalized mode 
propagation in first-order optical systems with loss or gain," J. Opt. Soc. Am. 72, 1409-1420 (1982).

9. H. M. Ozaktas, B. Barshan, D. Mendlovic, and L. Onural, "Convolution, filtering, and multiplexing in fractional Fourier domains and their relation to chirp and wavelet transforms," J. Opt. Soc. Am. A 11, 547-559 (1994).
10. A. W. Lohmann, "Image rotation, Wigner rotation, and the fractional Fourier transform," J. Opt. Soc. Am. A 10, 21812186 (1993).

11. H. M. Ozaktas and D. Mendlovic, "Fractional Fourier transform as a tool for analyzing beam propagation and spherical mirror resonators," Opt. Lett. 19, 1678-1680 (1994). 